\section{Electroencefalograma cuantitativo en la predicción precoz de la isquemia cerebral tardía en hemorragia subaracnoidea aneurismática. Reporte de un caso}

\author{
JOSÉ LUIS CASTILLO ${ }^{1,2}$, PABLO REYES S. ${ }^{1,2,3, \mathrm{a}}$, \\ RODRIGO GUTIÉRREZ ${ }^{4,5, a}$, JOSÉ MANUEL MATAMALA ${ }^{2,6,7, a}$, \\ LUIS SEGOVIA ${ }^{1}$, PEDRO MENENDEZ ${ }^{1}$, \\ CATALINA TORRES ${ }^{1,2}$, RICARDO CID $^{8, b}$, \\ GONZALO BUSTAMANTE ${ }^{1,2,3}$, WALTER FEUERHAKE ${ }^{1,2,3}$
}

\section{Quantitative electroencephalogram for prediction of delayed ischemia in subarachnoid haemorrhage. Report of one case}

Subarachnoid hemorrhage ( $S A H$ ) is a devastating disease, with a mortality rate of $35 \%$. Among patients who survive the initial bleeding, the leading cause of morbidity and mortality is delayed cerebral ischemia (DCI). Electroencephalography (EEG) can detect cerebral ischemia in the early stages. We report a 66-year-old female patient who consulted for ictal headache and impaired consciousness. On admission, she was confused, dysarthric, and with meningeal signs. Brain angio-CT showed SAH FISHER IV and an aneurysm of the left posterior cerebral artery. After excluding the aneurysm (by coiling), the patient recovered the altered consciousness. Continuous EEG monitoring was initiated. On the sixth day of follow up, she had a transient headache and apathy. The brain MRI showed low cerebral blood flow in the left frontotemporal area, without ischemic lesions. On the seventh day, she presented expression aphasia and right facial-brachial paresis. Angiography confirmed severe vasospasm in M1 and M2 segments bilaterally. Pharmacological angioplasty with nimodipine was performed, with an excellent radiological response, although not clinical. A second MRI was carried out on the eighth day, which showed a left insular infarction and generalized vasospasm. A second therapeutic angiography was performed; the patient persisted with aphasia and left central facial paresis. The quantitative EEG analysis performed retrospectively showed a generalized reduction in the spectral edge frequency 95 (SEF95; meaning slowing in the EEG signal) at the fourth day of follow up, three days earlier than the clinical and imaging diagnosis of DCI was established.

(Rev Med Chile 2021; 149: 1377-1381)

Key words: Brain Ischemia; Electroencephalography; Subarachnoid Hemorrhage.
'Servicio de Neurología,

Neurocirugía y Neurorradiología intervencional, Clínica Santa

María. Santiago, Chile.

${ }^{2}$ Departamento de Ciencias

Neurológicas Oriente, Facultad de Medicina, Universidad de Chile.

Santiago, Chile.

${ }^{3}$ Corporación de Investigación de Neurología de Santiago

(CINSAN).

${ }^{4}$ Centro de Investigación Clínica Avanzada, Hospital Clínico de la Universidad de Chile. Santiago,

Chile.

${ }^{5}$ Departamento de Anestesiología y Medicina Perioperatoria,

Facultad de Medicina

Universidad de Chile. Santiago, Chile.

${ }^{6}$ Departamento de Neurociencia,

Facultad de Medicina,

Universidad de Chile. Santiago,

Chile.

${ }^{7}$ Instituto Milenio de Neurociencia Biomédica (BNI), Facultad de Medicina, Universidad de Chile. Santiago, Chile.

${ }^{8}$ RCtec Cadwell Chile. Santiago, Chile.

aPhD.

bIngeniero.

Trabajo recibió apoyo financiero de la Dirección Académica de Clínica Santa María. Esta institución no tuvo influencias en el diseño, ni análisis del caso. Tampoco en la preparación, revisión ni aprobación del manuscrito.

Los autores declaran no tener conflictos de interés.

Recibido el 27 de junio de 2020, aceptado el 12 de octubre de 2021

Correspondencia a: Dr. José Luis Castillo José Manuel Infante 553, Providencia. Santiago, Chile. jlcastillo57@gmail.com 
L a hemorragia subaracnoidea aneurismática (HSAa) es una enfermedad devastadora, rebovasculares $(\mathrm{ACV})^{1}$. El 35\% de los pacientes que sufren una HSAa muere ${ }^{2}, 15 \%$ sobrevive con secuelas graves y $20-35 \%$ tienen moderada o buena recuperación $\mathrm{n}^{2,3}$.

Las principales causas neurológicas de morbimortalidad son el resangrado y la isquemia cerebral tardía (ICT). La ICT se define como el deterioro neurológico o la caída en la escala de Glasgow $>2$ puntos por más de 1 hora que no puede ser explicada por otra causa ${ }^{3}$. La ICT ocurre en $1 / 3$ de los pacientes con HSAa, habitualmente entre los días 4 y 14 tras el sangrado y es su complicación más grave $e^{4,5}$.

El electroencefalograma (EEG) es el registro de la actividad eléctrica cerebral ${ }^{6}$. El EEG puede detectar precozmente la isquemia ${ }^{6,7}$. Los cambios electroencefalográficos son aparición de actividad delta polimorfa y atenuación de la actividad rápida. El análisis de estos cambios se facilita con el monitoreo EEG continuo (EEGc) y el análisis cuantitativo $(\mathrm{EEGq})^{8,9}$. El EEGq es una técnica que combina la compresión de tiempo y reducción de canales para analizar la amplitud, frecuencias, simetría, ritmo y continuidad del $\mathrm{EEGc}^{9,10}$. Esto permite analizar cambios o tendencias en horas a días, según la presencia de distintos rangos de frecuencia (potencia). No hay evidencia concluyente respecto a cuáles son los parámetros del EEGq más útiles para la identificación de la ICT, aunque el aumento de las potencias en rangos theta y delta y la disminución de la potencia de rangos alfa y beta son los indicadores más promisorios ${ }^{11}$. Uno de los parámetros cuantitativos derivado del EEG es la frecuencia de borde espectral (SEF, spectral edge frequency, por su sigla en inglés). Esta es una medida ampliamente utilizada para el análisis y procesamiento de señales ${ }^{12}$. Los SEFx denotan la frecuencia bajo la cual se encuentra el x porcentaje del poder total de la señal en cuestión. En condiciones de enlentecimiento global de la actividad electroencefalográfica, en las que la potencia relativa de las bandas lentas delta aumenta, el SEF95 disminuye, y si predominan las frecuencias rápidas, aumenta

Se presenta el caso de una paciente con HSAa que presentó cambios significativos en el EEG continuo y EEGq, (SEF), antes del diagnóstico clínico e imagenológico de ICT.

\section{Caso clínico}

Mujer de 66 años, con antecedentes de tabaquismo y obesidad. Consultó por cefalea ictal, seguido de compromiso de conciencia. Al ingreso se encontraba somnolienta, confusa, disártrica y con signos meníngeos presentes (WFNS II). Se realizó AngioTC de cerebro que evidenció HSA extensa (FISHER IV), la presencia de un aneurisma de la arteria cerebral posterior izquierda de $2 \times 3 \mathrm{~mm}$ y moderada hidrocefalia. Se instaló un drenaje ventricular externo y luego coiling al aneurisma. Evolucionó con recuperacion completa del compromiso de conciencia y una leve paresia facial central izquierda. Al día siguiente se instaló monitoreo EEG continuo.

El sexto día de evolución presentó cefalea y apatía transitoria. Se realizó una resonancia magnética (RM) de cerebro que demostró HSA y una pequeña área de enlentecimiento en secuencias de perfusión (PWI) frontotemporal izquierdo, sin lesiones isquémicas. El 7o día de evolución, la paciente presentó afasia de expresión y paresia faciobraquial derecha. Se realizó angiografía que evidenció vasoespasmo grave de la arteria cerebral media (ACM) izquierda en segmentos M1 y M2, realizándose angioplastía farmacológica con nimodipino, con buena respuesta radiológica. Tras el procedimiento persistió la afasia de expresión y recuperó la paresia derecha. El $8^{\circ}$ día se realizó una segunda RM, que mostró un infarto insular izquierdo, vasoespasmo de ambos sifones carotídeos, ACM M1, cerebral anterior A1, cerebral posterior P1 izquierdas y extensas áreas de enlentecimiento frontal, parietal y temporal izquierdas en PWI (Figura 1). El mismo día se realizó angioplastía mecánica y farmacológica, evolucionando sin nuevo deterioro neurológico, persistiendo con afasia de expresión y paresia facial central izquierda. Dos días después (9º día) la RM demostró una nueva lesión isquémica frontoparietal izquierda y disminución del vasoespasmo. Actualmente solo con afasia.

El registro y análisis del EEG se realizó con un montaje longitudinal bipolar (sistema 10-20), con una tasa de muestreo de $250 \mathrm{~Hz}$. El análisis del EEGc fue ciego en relación a la evolución clínica y de laboratorio. Se mantuvo normal hasta el quinto día de evolución, cuando apareció actividad lenta focal frontal derecha intermitente. Posteriormente, en los días 6 y 7, el EEGc se hizo lento bilateral, para hacerse constante a partir del 
octavo día de evolución. No se detectó actividad epileptiforme ni otros hallazgos. Para el análisis del EEGq se calculó SEF95 utilizando MATLAB y herramientas multitaper implementadas en Chronux. Cada 2 minutos de registro, se evaluó si la señal se encontraba libre de ruido eléctrico, en cuyo caso se calculó el respectivo SEF95. Para la construcción de mapa de colores se promediaron todos los valores de SEF95 obtenidos en cada día. En la Figura 2, se presentan mapas de colores (topoflot) de los valores del SEF95. El cuarto día de evolución, 3 días antes del empeoramiento clínico y un día antes de la observación de actividad lenta en el registro del EEGc, se observó una importante disminución del SEF95 en forma generalizada (enlentecimiento de la señal EEG), que se mantuvo los días sucesivos. El análisis cuantitativo del EEG calculando los parámetros variabilidad del ritmo alfa y la relación alfa/delta no fueron concluyentes.

La paciente autorizó la publicación de su caso.

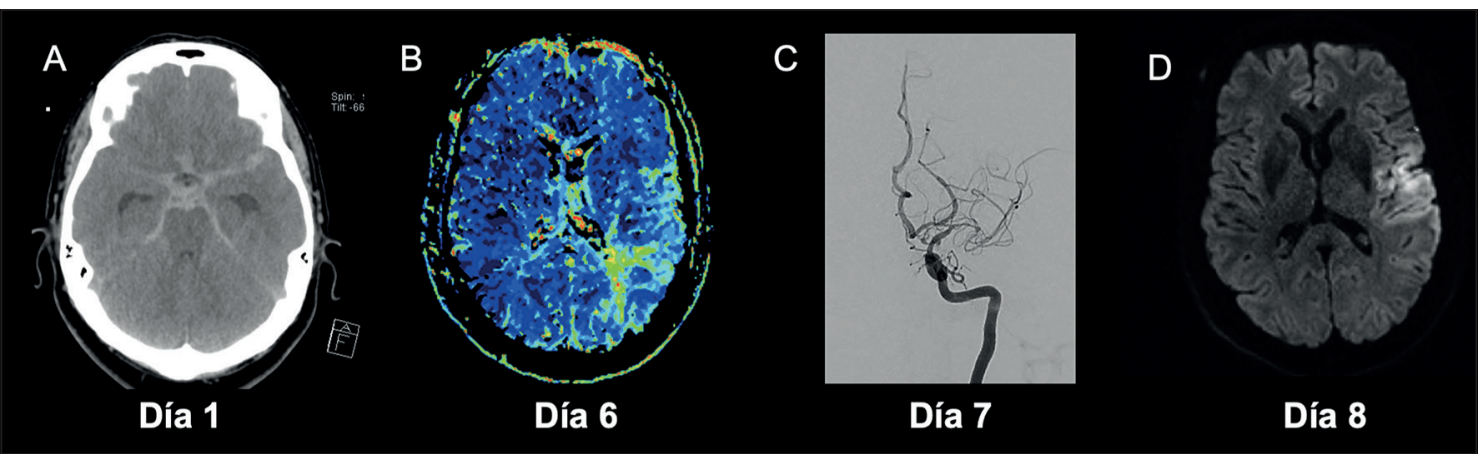

Figura 1. A: TC de cerebro, que muestra HSA difusa al momento del diagnóstico. B: Al $6^{\circ}$ día de evolución la paciente presentó cefalea y apatía transitorias por lo que se solicita RM de cerebro, que muestra hipoperfusión parietal izquierda, sin infarto en la difusión (no mostrado). C: Al día $7^{\circ}$ presentó afasia y hemiparesia derecha, se realiza angiografía cerebral de urgencia, que muestra vasoespasmo intenso a nivel silviano izquierdo. D: Al día $8^{\circ}$ se realiza RM de cerebro de control, que evidencia infarto fronto insular izquierdo.

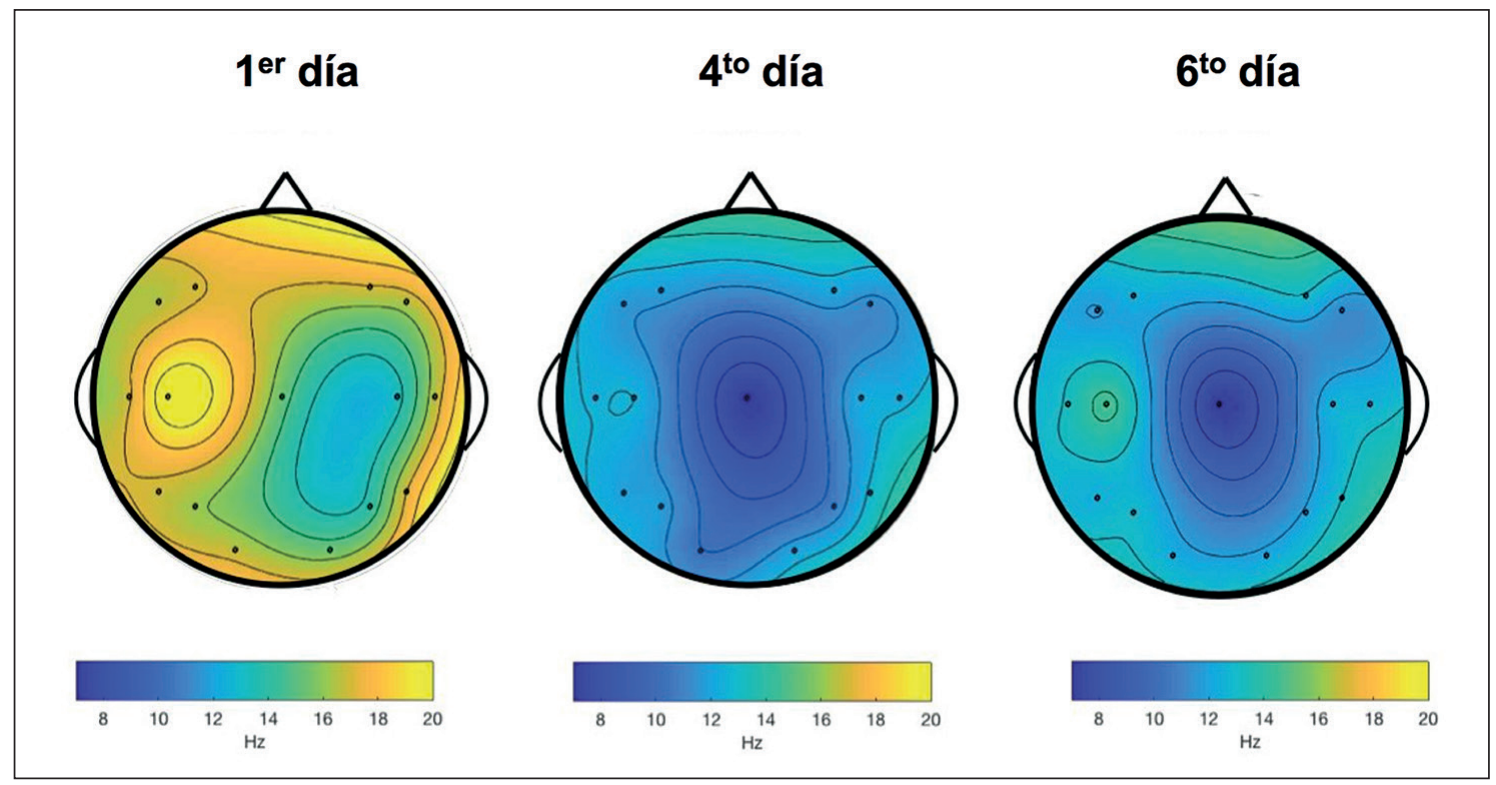

Figura 2. Mapas del SEF95 los días 1, 4 y 6 de evolución. El cuarto día se observa una disminución del SEF (color azul) debido al aumento en la potencia de las ondas lentas del espectro, principalmente delta. 


\section{Discusión}

En nuestra paciente con HSAa, el EEGc reveló actividad lenta bilateral ocasional el día anterior al deterioro clínico. El SEF95 en el EEGq demostró un empeoramiento bilateral permanente 3 días antes de la aparición de nueva focalidad y mientras la PWI por RNM aún no demostraba alteraciones sustantivas.

La ICT es la principal causa de morbimortalidad en pacientes con HSAa una vez tratado el aneurisma, y si bien puede acompañarse de vasoespasmo, este no es requisito ${ }^{13}$. Este caso ejemplifica cómo el analisis de variables cuantitativas del EEGc, especificamente el SEF95, es capaz de predecir hasta $72 \mathrm{~h}$ antes el inicio clinico de la ICT.

La presencia de alteraciones bilaterales en el EEGq y la caída bilateral en el SEF95 contrastan con la clínica focal hemisférica izquierda y la lateralización en la PWI, esto apoya que la fisiopatología de la ICT no depende solo de fenómenos vasculares y traduce disfunción eléctrica cortical que precede al desarrollo de la isquemia definitiva. Entre los eventos responsables de la ICT se propone el desarrollo de actividad epileptiforme y spreading depolarization que podrían aumentar las demandas metabólicas y contribuir a la isquemia $^{14}$. Estos cambios eléctricos podrían alterar el EEG y preceder a los cambios imagenológicos. Probablemente, la isquemia se produce en los territorios vasculares más alterados o con menor circulación colateral.

Claassen et al., en el año 2004, publicaron los primeros resultados del EEGq en la detección de ICT en 34 pacientes con HSA grave ${ }^{11}$, donde el $26 \%$ de los pacientes sufrió ICT y los cambios en el EEGq antecedieron en 6 a 24 h a los síntomas e imágenes. Gollwitzer et al. encontraron una fuerte asociación entre la disminución de la potencia del alfa y la posterior aparición de vasoespasmo e $\mathrm{ICT}^{15}$. Una revisión sistemática de 18 estudios $^{16}$, con 481 pacientes con HSAa, concluyó que el EEGc detecta un número importante de crisis epilépticas subclínicas (7-18\%) y status no convulsivo (3-13\%) y sin asociación a complicaciones. Además, puede predecir la ICT en varias horas, aunque no es claro el beneficio clínico, ya que no se demostró una disminución de la morbimortalidad. Recientemente, Rosenthal et al. reportaron una serie de 103 pacientes estudiados prospectivamente. El EEGq predijo la ICT $24 \mathrm{~h}$ antes de los síntomas en la mayoría de los casos, con una sensibilidad mayor a $90 \%$ para predecir ICT y una especificidad de alrededor de $80 \%{ }^{17}$. Para la obtención de la sensibilidad y especificidad usaron la relación alfa/delta, la variabilidad alfa/delta del EEGq y la presencia de enlentecimientos focales, descargas epileptiformes y patrones periódicos. El deterioro en la variabilidad del ritmo alfa destacó por presentar muy pocos falsos positivos (2\%) y el más alto de los OR $(36,7)$. El número de pacientes que deberían ser monitorizados para predecir un caso de ICT varía entre 3 a 7.

En nuestra paciente, la variabilidad del ritmo alfa y la relación alfa/delta no fueron suficientemente consistentes. En cambio el SEF, que analiza todas las frecuencias de la señal EEG, mostró anormalidades tres días antes de que la paciente experimentara el deterioro clínico e imagenológico.

Entre otras ventajas, el EEG permite monitorizar en forma continua y no invasiva a pacientes críticos. Sin embargo, es difícil solo con los cambios de la señal "cruda" del EEG emitir una alerta al clínico sobre el eventual desarrollo de ICT. En cambio, el EEGq permite optimizar la evaluación del EEG al mostrar tendencias cuantitativas más permanentes.

Probablemente, la falta de un parámetro consistente en el EEGq y la escasa difusión de este tipo de análisis electroencefalográfico ha limitado su uso en nuestro medio.

La principal limitación de este reporte es que se trata de un solo caso. Esperamos que el análisis de un número mayor de pacientes nos permita avanzar en la determinación de la utilidad de los diferentes parámetros del EEGq y conocer el rol de otros factores, como el de la actividad epileptogénica, en la detección de ICT.

Los autores agradecen el apoyo y asesoría de E.U. Andrea Ampuero en el desarrollo y culminación de este caso clínico.

\section{Referencias}

1. Brown RD Jr, Broderick JP. Unruptured intracranial aneurysms: epidemiology, natural history, management options, and familial screening. Lancet Neurol. 2014; 13: 393-404.

2. Nieuwkamp DJ, Setz LE, Algra A, Linn FH, de Rooij NK, Rinkel GJ. Changes in case fatality of aneurysmal subarachnoid haemorrhage over time, according to age, 
sex, and region: a meta-analysis. Lancet Neurol. 2009; 8: 635-42.

3. Taufique Z, May T, Meyers E, Falo C, Mayer S, Agarwal $S$ et al. Predictors of poor quality of life 1 year after subarachnoid hemorrhage. Neurosurgery 2016; 78: 256-64.

4. Rowland MJ, Hadjipavlou G, Kelly M, Westbrook J, Pattinson KT. Delayed cere- bral ischaemia after subarachnoid haemorrhage: looking beyond vasospasm. $\mathrm{Br}$ J Anaesth. 2012; 109: 315-29.

5. Kassell NF, Torner JC, Haley EC Jr, Jane JA, Adams HP, Kongable GL. The Inter- national Cooperative Study on the Timing of Aneurysm Surgery. 1. Overall management results. J Neurosurg. 1990; 73: 18-36.

6. Amzica FL da SF. Cellular substrates of brain rhytms. In: Niedermeyer, editor. Electroencephalography basic principles, clinical applicatons and related fields. Lippincott Willams \& Wilkins, 2010; 33-64.

7. Hossmann KA. Viability thresholds and the penumbra of focal ischemia. Ann. Neurol. 1994; 36 (4): 557-65.

8. Herman ST, Abend NS, Bleck TP, Chapman KE, Drislane FW, Emerson RG, et al. Consensus statement on continuous EEG in critically ill adults and children, Part I: Indications. J Clin Neurophysiol. 2015; 32 (2): 87-95.

9. Swisher CB, Shah D, Sinha SR, Husain AM. Baseline EEG Pattern on Continuous ICU EEG Monitoring and Incidence of Seizures. J Clin Neurophysiol. 2015; 32 (2): 147-51.

10. Claassen J, Mayer SA, Hirsch LJ. Continuous EEG monitoring in patients with subarachnoid hemorrhage. J Clin Neurophysiol. 2005; 22 (2): 92-8.
11. Claassen J, Hirsch LJ, Kreiter KT, Du EY, Connolly ES, Emerson RG, Mayer SA. Quantitative continuous EEG for detecting delayed cerebral ischemia in patients with poor-grade subarachnoid hemorrhage. Clin Neurophysiol. 2004; 115 (12): 2699-710.

12. Schwender D, Dauderer M, Mulzen S, Klasing S, Finsterer U, Peter K. Spectral edge frequency of the electroencephalogram to monitor "depth" of anaesthesia with isoflurane or propofol. Br J Anesth 1996; 77: 179-84.

13. Hijdra A, Van Gijn J, Stefanko S, Van Dongen KJ, Vermeulen M, Van Crevel H. Delayed cerebral ischemia after aneurysmal subarachnoid hemorrhage: clinicoanatomic correlations. Neurology. 1986; 36: 329-33.

14. Weir B, Macdonald RL, Stoodley M. Etiology of cerebral vasospasm. Acta Neurochir Suppl. 1999; 72: 27-46.

15. Gollwitzer S, Groemer T, Ramppp S, Hagge M, Olmes $\mathrm{D}$, Huttner HB, et al. Early prediction of delayed cerebral ischemia in subarachnoid hemorrhage based on quantitative EEG: A prospective study in adults. Clin Neurophysiol. 2015; 126: 1514-23.

16. Kondziella D, Friberg CK, Wellwood I, Reiffurth C, Fabricius M, Dreier JP. Continuous EEG Monitoring in Aneurysmal Subarachnoid Hemorrhage: A Systematic Review. Neurocrit Care 2015; 22 (3): 450-61.

17. Rosenthal ES, Biswal S, Zafar SE, O Connor KL, Becheck $S$, Shenov AV, et al. Continuous electroencephalography predicts delayed cerebral ischemia after subarachnoid hemorrhage: A prospective study of diagnostic accuracy. Ann Neurol. 2018; 83 (5): 958-69. 